\title{
A CHARACTERIZATION OF HEREDITARY RINGS OF FINITE REPRESENTATION TYPE
}

\author{
BY PIOTR DOWBOR AND DANIEL SIMSON
}

In this note we announce a characterization of hereditary rings $R$ of finite representation type in terms of a sequence of partial Coxeter functors associated to $R$.

The methods we use are theory of almost split sequences [1], partial Coxeter functors [2], [3], [5], representations of species [7], and some ideas of Ringel $[8, \S 6]$.

We recall from [7] that a species $M=\left(F_{i},{ }_{i} M_{j}\right)_{i, j \in I}$ is a finite set of division rings $F_{i}$ and $F_{i}-F_{j}$ bimodules ${ }_{i} M_{j}$. Throughout we will suppose $M$ is a species such that ${ }_{i} M_{j} \neq 0$ implies $M_{i}=0$ for $i \neq j$. $M$ is called finite dimensional if the dimensions

$$
d_{i j}=\operatorname{dim}\left({ }_{i} M_{j}\right)_{F_{j}}, \quad d_{j i}=\operatorname{dim}_{F_{i}}\left(M_{i}\right)
$$

are finite for $i \neq j$. We denote by $r(M)$ the category of all finite dimensional right representations of $M$ (see [6], [8]). From $M$ we derive an oriented valued graph $(\Gamma, \mathbf{d}, \Omega)$ (not necessarily symmetrizable) with valued edges

$$
\stackrel{\left(d_{i j}, d_{j i}\right)}{\longrightarrow} \text {; }
$$

precisely when ${ }_{i} M_{j} \neq 0$. Given source (resp. sink) $k$ in $(\Gamma, \mathbf{d}, \Omega)$ we define a species $M^{k}=\left(F_{i},{ }_{i} N_{j}\right)_{i, j \in I}$ by taking

$$
{ }_{i} N_{j}=\left\{\begin{array}{l}
{ }_{k} M_{i}^{i}=\operatorname{Hom}_{F_{i}}\left({ }_{k} M_{i}, F_{i}\right) \text { for } j=k \quad\left(\text { resp. }={ }_{j} M_{k}^{j} \text { for } i=k\right) \\
\left.{ }_{i} M_{j} \text { for } j \neq k \quad \text { (resp. for } i \neq k\right) .
\end{array}\right.
$$

If $M$ is a finite dimensional species and $k$ is a sink in $(\Gamma, \mathbf{d}, \Omega)$ we can define a pair of Coxeter functors

having following properties:

$$
r(M) \underset{S_{k}^{-}}{\stackrel{S_{k}^{+}}{\rightleftarrows}} r\left(M^{k}\right)
$$

(c) $S_{k}^{+}$is left adjoint to $S_{k}^{+}$.

(c) Suppose $\mathbf{X}=\left(X_{i}, j^{\varphi_{i}}\right)$ is indecomposable in $r(M)$. Then

(i) $S_{k}^{+} \mathbf{X}=0$ if and only if $\mathbf{X} \cong \mathbf{F}_{k}$, where $\mathbf{F}_{k}$ has $F_{k}$ on $k$ th coordinate and zeros otherwise,

Received by the editors June 11, 1979.

AMS (MOS) subject classifications (1970). Primary 16A64; Secondary 16A46.

Key words and phrases. Species, Coyeter functors, ring of finite representation type. (c) 1980 American Mathematical Society 0002-9904/80/0000-0102/\$01.75 
(ii) if $S_{k}^{+} \mathbf{X}=0$ then there is a natural isomorphism $S_{k} S_{k}^{+} \mathbf{X} \simeq \mathbf{X}$ and $\operatorname{dim} S_{k}^{+} \mathbf{X}=s_{k}(\operatorname{dim} \mathbf{X})$ where $\operatorname{dim} \mathbf{X}=\left(\operatorname{dim}\left(X_{i}\right)_{F_{i}}\right)_{i \in I}$ and $s_{k}: Z^{\Gamma} \rightarrow Z^{\Gamma}$ is the corresponding reflection defined by using the matrix $\left(d_{i j}\right)$.

$\left(\mathbf{c}_{3}^{+}\right)$If $\mathbf{X}$ and $\mathbf{Y}$ are indecomposables in $r(M)$ and $S_{k}^{+} \mathbf{X} \neq 0, S_{k}^{+} \mathbf{Y} \neq 0$ then there is a natural isomorphism $\operatorname{Hom}(\mathbf{X}, \mathbf{Y}) \cong \operatorname{Hom}\left(S_{k}^{+} \mathbf{X}, S_{k}^{+} \mathbf{Y}\right)$.

$\left(\mathbf{c}_{4}^{+}\right)$If $\mathbf{0} \rightarrow \mathbf{X} \rightarrow \mathbf{Y} \rightarrow \mathbf{Z} \rightarrow 0$ is an almost split sequence in $r(M)$ with $\mathbf{X}$ noninjective and $S_{k}^{+} \mathbf{X} \neq 0$ then $0 \rightarrow S_{k}^{+} \mathbf{X} \rightarrow S_{k}^{+} \mathbf{Y} \rightarrow S_{k}^{+} \mathbf{Z} \rightarrow 0$ is almost split.

The properties $\left(\mathrm{c}_{1}^{+}\right)-\left(\mathrm{c}_{4}^{+}\right)$and analogous properties $\left(\mathbf{c}_{1}^{-}\right)-\left(\mathbf{c}_{4}^{-}\right)$for the case $k$ is a source can be found in [2], [5].

Now let $M$ be a finite dimensional species, $(\Gamma, d, \Omega)$ its valued graph and $k_{1}, \ldots, k_{n}$ a fixed admissible orientation (see [5]). We define a sequence $\left\{k_{j}^{\prime}\right\}_{j \in Z}$, where $Z$ is the set of integral numbers. We put

$$
k_{j}^{\prime}= \begin{cases}k_{r+1} & \text { if } j \geqslant 0, j=t n+r, 0 \leqslant r<n, \\ k_{n-r+1} & \text { if } j<0,-j=t n+r, 0 \leqslant r<n .\end{cases}
$$

Moreover for $m \in Z$ we define inductively species $M^{(m)}$ putting $M^{(0)}=M$ and

$$
M^{(m)}= \begin{cases}\left(M^{(m-1)}\right)^{k_{m-1}^{\prime}} & \text { for } m \geqslant 1, \\ \left(M^{(m+1)}\right)^{k_{m}^{\prime}} & \text { for } m \leqslant-1 .\end{cases}
$$

We say that $M$ has finite dimension property if all species $M^{(m)}, m \in Z$, are finite dimensional. In this case we denote by $\left(\Gamma, \mathrm{d}^{m}, \Omega^{m}\right)$ the valued graph of the species $M^{(m)}$ and by $s_{m}: Z^{\Gamma} \rightarrow Z^{\Gamma}$ the reflection $s_{k_{m}^{\prime}}$ defined by using the matrix $\mathrm{d}^{m}=\left(d_{i j}^{m}\right)$. Following Ringel [8] we say that $M$ has the constant dimension property if $d_{i j}^{m}=d_{i j}$ for all $i, j \in I, m \in Z$.

Now given a species $M$ with the finite dimension property we have an infinite diagram

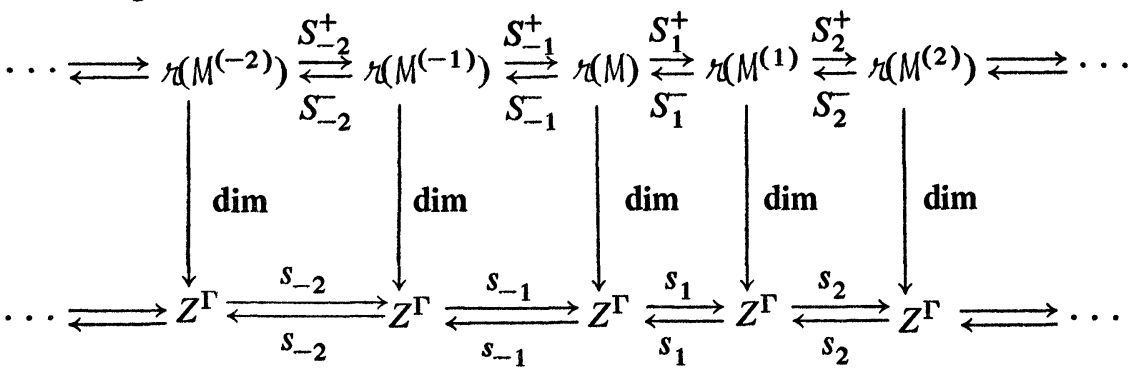

where $S_{i}^{+}$and $S_{i}^{-}$are appropriate partial Coxeter functors and $s_{i}$ the corresponding reflections. The diagram will be called the infinite Coxeter scheme of $M$ with respect to the admissible sequence $k_{1}, \ldots, k_{n}$. Now we are able to formulate the main result of the paper.

THEOREM. Let $M$ be a finite dimensional species and suppose that its valued graph $(\Gamma, \mathbf{d}, \Omega)$ is connected. The category $r(M)$ is of finite representation type if and only if $M$ has the finite dimension property and there exists a 
number $m>0$ such that $s_{m} \cdots s_{1}\left(e_{i}\right) \ngtr 0$ for any source $i$ of $\Gamma$, where $e_{i} \in$ $Z^{\Gamma}$ is the vector having 1 on the $i$-th coordinate and zeros otherwise. Moreover, if $m$ is minimal with the above property then

(a) The mapping dim: $r(M) \rightarrow Z^{\Gamma}$ is a one-one correspondence between isomorphism classes of indecomposable representations in $r(M)$ and vectors in $Z^{\Gamma}$ of the form $s_{1} \cdots s_{t-1}\left(e_{k_{t}^{\prime}}\right)$ where $t<m$ and $k_{t}^{\prime}$ is a sink in $\left(\Gamma, \mathbf{d}^{t}, \Omega^{t}\right)$. In other words any indecomposable representation $\mathbf{X}$ in $r(M)$ has the form $\mathbf{X}$ $\cong S_{1}^{-} \cdots S_{t-1}^{-} \mathbf{F}_{k_{t}^{\prime}}$, where $t<m$ and $\mathbf{F}_{k_{t}^{\prime}}$ is a simple projective in $r_{\left(M^{(t-1)}\right)}$ with $F_{k_{t}^{\prime}}$ on the $k_{t}^{\prime}$ th coordinate and zeros otherwise.

(b) There are equivalences $r\left(M^{(i)}\right) \approx r\left(M^{\left(m^{\prime} q+i\right)}\right)$ for $i=0,1, \ldots, m^{\prime}-1$ and $q \in Z$.

Observe that the theorem gives us a characterization of hereditary rings $R$ of finite representation type having the property that $R$ are generated over their centers by a set of cardinality $\aleph_{\iota}$, the first strongly inaccessible cardinal number. Indeed, if $R$ is such a ring and $M_{R}$ is the species of $R$ then by [9, Corollary 4.6] there is an equivalence mod- $R \simeq{ }_{r}\left(M_{R}\right)$.

Let us recall from [6] that if $M$ is finite dimensional and the division rings $F_{i}, i \in I$, are finitely generated over their centers then $M$ has the constant dimension property and therefore $\Omega(M)$ is of finite representation type if and only if its valued graph is a Dynkin diagram.

REMARK. There exists a hereditary ring $R$ of infinite representation type such that its valued graph $(\Gamma, \mathbf{d}, \Omega)$ is the Dynkin diagram

(apply [2] and [4]).

$$
\stackrel{(1,2)}{\longrightarrow} \text {. }
$$

\section{REFERENCES}

1. M. Auslander and I. Reiten, Representation theory of Artin algebras. III, Almost split sequences, Comm. Algebra 3 (1975), 239-294.

2. M. Auslander, M. I. Platzeck and I. Reiten, Coxeter functors without diagrams, Trans. Amer. Math. Soc. 250 (1979) 1-46.

3. I. N. Berstein, I. M. Gelfand and V. A. Ponomarev, Coxeter functors and Gabriel's theorem, Uspechi Mat. Nauk 28 (1973), 19-33.

4. P. M. Cohn, Quadratic extensions of skew fields, Proc. London Math. Soc. (3) 11 (1961), 531-556.

5. V. Dlab and C. M. Ringel, Representations of graphs and algebras, Mem. Amer. Math. Soc. No. 173 (1976).

6. P. Dowbor and D. Simson, Quasi-Artin species and rings of finite representation type, J. Algebra (to appear).

7. P. Gabriel, Indecomposable representations. II, Symposia Mathematica, vol. 11, Academic Press, London. 1973, pp. 81-104. 269-302.

8. C. M. Ringel, Representations of K-species and bimodules, J. Algebra 41 (1976),

9. D. Simson, Categories of representations of species, J. Pure Appl. Algebra 14 (1979), $101-114$.

INSTITUTE OF MATHEMATICS, NICHOLAS COPERNICUS UNIVERSITY, 87-100 TORUN', POLAND 